\title{
Axiomatizing Tropical Semirings
}

\author{
Luca Aceto $^{1}$, Zoltán Ésik ${ }^{2 \star}$, and Anna Ingólfsdóttir ${ }^{1}$ \\ 1 BRICS $^{\star \star \star}$, Department of Computer Science, Aalborg University, \\ Fredrik Bajers Vej 7-E, DK-9220 Aalborg Ø, Denmark. \\ 2 Department of Computer Science, \\ A. József University, Árpád tér 2, 6720 Szeged, Hungary.
}

\begin{abstract}
This paper studies the equational theory of various exotic semirings presented in the literature. Exotic semirings are semirings whose underlying carrier set is some subset of the set of real numbers equipped with binary operations of minimum or maximum as sum, and addition as product. Two prime examples of such structures are the $(\max ,+)$ semiring and the tropical semiring. It is shown that none of the exotic semirings commonly considered in the literature has a finite basis for its equations, and that similar results hold for the commutative idempotent weak semirings that underlie them. For each of these commutative idempotent weak semirings, the paper offers characterizations of the equations that hold in them, explicit descriptions of the free algebras in the varieties they generate, and relative axiomatization results.
\end{abstract}

Keywords and Phrases: Equational logic, varieties, complete axiomatizations, relative axiomatizations, commutative idempotent weak semirings, tropical semirings, convexity, exponential time complexity.

\section{Introduction}

Exotic semirings, i.e., semirings whose underlying carrier set is some subset of the set of real numbers $\mathbb{R}$ equipped with binary operations of minimum or maximum as sum, and addition as product, have been invented and reinvented many times since the late fifties in various fields of research. This family of structures consists of semirings whose sum operation is idempotent - two prime examples are the $(\max ,+)$ semiring $(\mathbb{R} \cup\{-\infty\}$, $\max ,+,-\infty, 0)$ (see [5] Chapter 3] for a general reference), and the tropical semiring $(\mathbb{N} \cup\{\infty\}$, min, $+, \infty, 0)$ introduced in [20]. (Henceforth, we shall write $\vee$ and $\wedge$ for the binary maximum and minimum operations, respectively.) Interest in idempotent semirings arose in the 1950 s through the observation that some problems in discrete optimization could be linearized over such structures (see, e.g., 22] for a survey). Since then, the study of idempotent semirings has forged productive connections with such diverse fields as, e.g., performance evaluation of manufacturing systems,

^ Partially supported by grant no. T30511 from the National Foundation of Hungary for Scientific Research.

$\star \star \star$ Basic Research in Computer Science.

F. Honsell and M. Miculan (Eds.): FOSSACS 2001, LNCS 2030, pp. 42-56 2001.

(C) Springer-Verlag Berlin Heidelberg 2001 
discrete event system theory, graph theory (path algebra), Markov decision processes, Hamilton-Jacobi theory, and automata and language theory (automata with multiplicities). The interested reader is referred to [10] for a survey of these more recent developments. Here we limit ourselves to mentioning some of the deep applications of variations on the tropical semiring in automata theory and the study of formal power series.

The tropical semiring $(\mathbb{N} \cup\{\infty\}, \wedge,+, \infty, 0)$ was originally introduced by Simon in his solution (see [20]) to Brzozowski's celebrated finite power property problem-i.e., whether it is decidable if a regular language $L$ has the property that, for some $m \geq 0$,

$$
L^{*}=1+L+\cdots+L^{m} .
$$

The basic idea in Simon's argument was to use automata with multiplicities in the tropical semiring to reformulate the finite power property as a Burnside problem. (The original Burnside problem asks if a finitely generated group must necessarily be finite if each element has finite order [7].) The tropical semiring was also used by Hashiguchi in his independent solution to the aforementioned problem [12, and in his study of the star height of regular languages (see, e.g., [13 14]). (For a tutorial introduction on how the tropical semiring is used to solve the finite power property problem, we refer the reader to [18].) The tropical semiring also plays a key role in Simon's study of the nondeterministic complexity of a standard finite automaton 21 .

The study of automata and regular expressions with multiplicities in the tropical semiring is by now classic, and has yielded many beautiful and deep results - whose proofs have relied on the study of further exotic semirings. For example, Krob has shown that the equality problem for regular expressions with multiplicities in the tropical semiring is undecidable 15. by introducing the equatorial semiring $(\mathbb{Z} \cup\{\infty\}, \wedge,+, \infty, 0)$, showing that the equality problem for it is undecidable, and finally proving that the two decidability problems are equivalent. Partial decidability results for certain kinds of equality problems over the tropical and equatorial semirings are studied in [16].

Another classic question for the language of regular expressions, with or without multiplicities, is the study of complete axiom systems for them (see, e.g., [9]). Along this line of research, Bonnier-Rigny and Krob have offered a complete system of identities for one-letter regular expressions with multiplicities in the tropical semiring [6]. However, to the best of our knowledge, there has not been a systematic investigation of the equational theory of the different exotic semirings studied in the literature. This is the aim of this paper.

Our starting points are the results we obtained in [1/2]. In [1] we studied the equational theory of the max-plus algebra of the natural numbers $\mathbf{N}_{\vee}=$ $(\mathbb{N}, \vee,+, 0)$, and proved that not only its equational theory is not finitely based, but, for every $n$, the equations in at most $n$ variables that hold in it do not form an equational basis. Another view of the non-existence of a finite basis for the variety generated by this algebra is offered in [2], where we showed that the collection of equations in two variables that hold in it has no finite equational axiomatization. 
The algebra $\mathbf{N}_{\vee}$ is an example of a structure that we call in this paper commutative idempotent weak semiring (abbreviated to ciw-semiring). Since ciw-semirings underlie many of the exotic semirings studied in the literature, we begin our investigations in this paper by systematically generalizing the results from 12 to the structures $\mathbf{Z}_{\vee}=(\mathbb{Z}, \vee,+, 0)$ and $\mathbf{N}_{\wedge}=(\mathbb{N}, \wedge,+, 0)$. Our initial step in the study of the equational theory of these ciw-semirings is the characterization of the (in)equations that hold in them (Propositions 3 and 4). These characterizations pave the way to explicit descriptions of the free algebras in the varieties $\mathcal{V}\left(\mathbf{Z}_{\vee}\right)$ and $\mathcal{V}\left(\mathbf{N}_{\wedge}\right)$ generated by $\mathbf{Z}_{\vee}$ and $\mathbf{N}_{\wedge}$, respectively, (Theorem 1) and yield finite axiomatizations of the varieties $\mathcal{V}\left(\mathbf{N}_{\vee}\right)$ and $\mathcal{V}\left(\mathbf{N}_{\wedge}\right)$ relative to $\mathcal{V}\left(\mathbf{Z}_{\vee}\right)$ (Theorem 21). We then show that, like $\mathcal{V}\left(\mathbf{N}_{\vee}\right)$, the varieties $\mathcal{V}\left(\mathbf{Z}_{\vee}\right)$ and $\mathcal{V}\left(\mathbf{N}_{\wedge}\right)$ are not finitely based. The non-finite axiomatizability of the variety $\mathcal{V}\left(\mathbf{Z}_{\vee}\right)$ (Theorem 3) is a consequence of the similar result for $\mathcal{V}\left(\mathbf{N}_{\vee}\right)$ and of its finite axiomatizability relative to $\mathcal{V}\left(\mathbf{Z}_{\vee}\right)$. The proof of the non-existence of a finite basis for the variety $\mathcal{V}\left(\mathbf{N}_{\wedge}\right)$ (Theorem [4 $)$ is more challenging, and relies on a model-theoretic construction. This construction yields that, as for $\mathcal{V}\left(\mathbf{N}_{\vee}\right)$, for every natural number $n$, the set of equations in at most $n$ variables that hold in $\mathcal{V}\left(\mathbf{N}_{\wedge}\right)$ does not form an equational basis for this variety. A similar strengthening of the non-finite axiomatizability result holds for the variety $\mathcal{V}\left(\mathbf{Z}_{\vee}\right)$, for which we also show that the collection of equations in two variables that hold in it does not have a finite axiomatization.

We then move on to study the equational theory of the exotic semirings presented in the literature that are obtained by adding bottom elements to the above ciw-semirings. More specifically, we examine the following semirings:

$$
\begin{aligned}
\mathbf{Z}_{\vee,-\infty} & =(\mathbb{Z} \cup\{-\infty\}, \vee,+,-\infty, 0), \\
\mathbf{N}_{\vee,-\infty} & =(\mathbb{N} \cup\{-\infty\}, \vee,+,-\infty, 0) \text { and } \\
\mathbf{N}_{\vee,-\infty}^{-} & =\left(\mathbb{N}^{-} \cup\{-\infty\}, \vee,+,-\infty, 0\right),
\end{aligned}
$$

where $\mathbb{N}^{-}$stands for the set of nonpositive integers. Since $\mathbf{Z}_{\vee,-\infty}$ and $\mathbf{N}_{\vee,-\infty}^{-}$ are easily seen to be isomorphic to the semirings $\mathbf{Z}_{\wedge, \infty}=(\mathbb{Z} \cup\{\infty\}, \wedge,+, \infty, 0)$ and $\mathbf{N}_{\wedge, \infty}=(\mathbb{N} \cup\{\infty\}, \wedge,+, \infty, 0)$, respectively, the results that we obtain apply equally well to these algebras. In fact, rather than studying the algebra $\mathbf{N}_{\vee}^{-},-\infty$, we work with the semiring $\mathbf{N}_{\wedge, \infty}$. (The semirings $\mathbf{Z}_{\wedge, \infty}$ and $\mathbf{N}_{\wedge, \infty}$ are usually referred to as the equatorial semiring [15] and the tropical semiring [20, respectively. The semiring $\mathbf{N}_{\vee,-\infty}$ is called the polar semiring in [17].) We offer non-finite axiomatizability results for the semirings $\mathbf{Z}_{\vee,-\infty}, \mathbf{N}_{\vee,-\infty}$ and $\mathbf{N}_{\wedge, \infty}$.

Throughout the paper, we shall use standard notions and notations from universal algebra that can be found, e.g., in [8. All the proofs of our technical results are omitted for lack of space.

A full version of this study, containing full proofs and many more results, including those presented in [3], can be found in [4]. 


\section{$2 \quad$ Background Definitions}

We begin by introducing some notions that will be used in the technical developments to follow.

A commutative idempotent weak semiring (henceforth abbreviated to ciwsemiring) is an algebra $\mathbf{A}=(A, \vee,+, 0)$ such that $(A, \vee)$ is an idempotent commutative semigroup, i.e., a semilattice, $(A,+, 0)$ is a commutative monoid, and such that addition distributes over the $\vee$ operation. Thus, the following equations hold in $\mathbf{A}$ :

$$
\begin{aligned}
x \vee(y \vee z) & =(x \vee y) \vee z \\
x \vee y & =y \vee x \\
x \vee x & =x \\
x+(y+z) & =(x+y)+z \\
x+y & =y+x \\
x+0 & =x \\
x+(y \vee z) & =(x+y) \vee(x+z) .
\end{aligned}
$$

A homomorphism of ciw-semirings is a function which preserves the $\vee$ and + operations and the constant 0 .

A commutative idempotent semiring is an algebra $(A, \vee,+, \perp, 0)$ such that $(A, \vee,+, 0)$ is a ciw-semiring which satisfies the equations

$$
\begin{aligned}
& x \vee \perp=x \\
& x+\perp=\perp .
\end{aligned}
$$

A homomorphism of commutative idempotent semirings also preserves $\perp$.

Suppose that $\mathbf{A}=(A, \vee,+, 0)$ is a ciw-semiring. Assume that $\perp \notin A$ and let $A_{\perp}=A \cup\{\perp\}$. Extend the operations $\vee$ and + given on $A$ to $A_{\perp}$ by defining

$$
\begin{aligned}
& a \vee \perp=\perp \vee a=a \\
& a+\perp=\perp+a=\perp,
\end{aligned}
$$

for all $a \in A_{\perp}$. We shall write $\mathbf{A}_{\perp}$ for the resulting algebra.

Lemma 1. For each ciw-semiring $\mathbf{A}$, the algebra $\mathbf{A}_{\perp}$ is a commutative idempotent semiring.

Remark 1. In fact, $\mathbf{A}_{\perp}$ is the free commutative idempotent semiring generated by $\mathbf{A}$.

Let $E_{c i w}$ denote the set of defining axioms of ciw-semirings, and $E_{c i}$ the set of axioms of commutative idempotent semirings. Moreover, let $\mathcal{V}_{\text {ciw }}$ denote the variety axiomatized by $E_{c i w}$.

In the remainder of this paper, we shall use $n x$ to denote the $n$-fold sum of $x$ with itself, and we take advantage of the associativity and commutativity of 
the operations. By convention, $n x$ stands for 0 when $n=0$. In the same way, the empty sum is defined to be 0 .

For each integer $n \geq 0$, we use $[n]$ to stand for the set $\{1, \ldots, n\}$, so that $[0]$ is another name for the empty set.

Lemma 2. With respect to the axiom system $E_{\text {ciw }}$ (respectively, $E_{c i}$ ), every term $t$ in the language of ciw-semirings (resp., commutative idempotent semirings), in the variables $x_{1}, \ldots, x_{n}$, may be rewritten in the form

$$
t=\bigvee_{i \in[k]} t_{i}
$$

where $k>0$ (resp., $k \geq 0$ ), each $t_{i}$ is a "linear combination"

$$
t_{i}=\sum_{j \in[n]} c_{i j} x_{j}
$$

and each $c_{i j}$ is in $\mathbb{N}$.

Terms of the form $\bigvee_{i \in[k]} t_{i}$, where each $t_{i}(i \in[k], k \geq 0)$ is a linear combination of variables, will be referred to as simple terms. When $k=0$, the term $\bigvee_{i \in[k]} t_{i}$ is just $\perp$. (Note that $k=0$ is only allowed for commutative idempotent semirings.) For any commutative idempotent (weak) semiring $\mathbf{A}$ and $a, b \in A$, we write $a \leq b$ to mean $a \vee b=b$. In any such structure, the relation $\leq$ so defined is a partial order, and the + and $\vee$ operations are monotonic with respect to it. Similarly, we say that an inequation $t \leq t^{\prime}$ between terms $t$ and $t^{\prime}$ holds in $\mathbf{A}$ if the equation $t \vee t^{\prime}=t^{\prime}$ holds. We shall write $\mathbf{A}=t=t^{\prime}$ (respectively, $\mathbf{A} \models t \leq t^{\prime}$ ) if the equation $t=t^{\prime}$ (resp., the inequation $t \leq t^{\prime}$ ) holds in $\mathbf{A}$. (In that case, we say that $\mathbf{A}$ is a model of $t=t^{\prime}$ or $t \leq t^{\prime}$, respectively.)

Definition 1. A simple inequation in the variables $x_{1}, \ldots, x_{n}$ is of the form

$$
t \leq \bigvee_{i \in[k]} t_{i},
$$

where $k>0$, and $t$ and the $t_{i}(i \in[k])$ are linear combinations of the variables $x_{1}, \ldots, x_{n}$. We say that the left-hand side of the above simple inequation contains the variable $x_{j}$, or that $x_{j}$ appears on the left-hand side of the simple inequation, if the coefficient of $x_{j}$ in $t$ is nonzero. Similarly, we say that the right-hand side of the above inequation contains the variable $x_{j}$ if for some $i$, the coefficient of $x_{j}$ in $t_{i}$ is nonzero.

Note that, for every linear combination $t$ over variables $x_{1}, \ldots, x_{n}$, the inequation $t \leq \perp$ is not a simple inequation.

Corollary 1. With respect to the axiom system $E_{\text {ciw }}$, any equation in the language of ciw-semirings is equivalent to a finite set of simple inequations. Similarly, with respect to $E_{c i}$, any equation in the language of commutative idempotent semirings is equivalent to a finite set of simple inequations or to an inequation of the form $x \leq \perp$ (in which case the equation has only trivial models). 
Henceforth in this study, we shall often abbreviate a simple inequation

$$
\sum_{j \in[n]} d_{j} x_{j} \leq \bigvee_{i \in[k]} \sum_{j \in[n]} c_{i j} x_{j}
$$

as $\bar{d} \leq\left\{\bar{c}_{1}, \ldots, \bar{c}_{k}\right\}$, where $\bar{d}=\left(d_{1}, \ldots, d_{n}\right)$ and $\bar{c}_{i}=\left(c_{i 1}, \ldots, c_{i n}\right)$, for $i \in[k]$. We shall sometimes refer to these inequations as simple $\vee$-inequations.

In the main body of the paper, we shall also study some ciw-semirings that, like the structure $\mathbf{N}_{\wedge}=(\mathbb{N}, \wedge,+, 0)$, have the minimum operation in lieu of maximum. The preliminary results that we have developed in this section apply equally well to these structures. Note that, for ciw-semirings of the form $(A, \wedge,+, 0)$, the partial order $\geq$ is defined by $b \geq a$ iff $a \wedge b=a$. In Defn. 1] we introduced the notion of simple $\vee$-inequation. Dually, we say that a simple $\wedge$-inequation in the variables $x_{1}, \ldots, x_{n}$ is an inequation of the form

$$
\bar{d} \cdot \bar{x} \geq \bigwedge_{i \in[k]} \bar{c}_{i} \cdot \bar{x}
$$

where $\bar{d}$ and the $\bar{c}_{i}(i \in[k])$ are vectors in $\mathbb{N}^{n}$. We shall often write

$$
\bar{d} \geq\left\{\bar{c}_{1}, \ldots, \bar{c}_{k}\right\}
$$

as a shorthand for this inequation.

\section{Min-Max-Plus Weak Semirings}

Our aim in this section will be to study the equational theory of the ciw-semirings that underlie most of the tropical semirings studied in the literature. More specifically, we shall study the following ciw-semirings:

$$
\mathbf{Z}_{\vee}=(\mathbb{Z}, \vee,+, 0), \mathbf{N}_{\vee}=(\mathbb{N}, \vee,+, 0) \text { and } \mathbf{N}_{\wedge}=(\mathbb{N}, \wedge,+, 0)
$$

equipped with the usual sum operation + , constant 0 and one of the operations $\checkmark$ for the maximum of two numbers and $\wedge$ for the minimum of two numbers. We shall sometimes use the fact that $\mathbf{Z}_{\vee}$ and $\mathbf{N}_{\wedge}$ are isomorphic to the ciw-semirings $\mathbf{Z}_{\wedge}=(\mathbb{Z}, \wedge,+, 0)$ and $\mathbf{N}_{\vee}^{-}=\left(\mathbb{N}^{-}, \vee,+, 0\right)$, respectively, where $\mathbb{N}^{-}$stands for the set of nonpositive integers.

Our study of the equational theory of these algebras will be based on the following uniform pattern. First, we offer a characterization of the simple inequations that hold in these ciw-semirings. These characterizations pave the way to concrete descriptions of the free algebras in the varieties generated by the algebras we study, and yield relative axiomatization results (Sect. [3.2). Finally we show that none of the ciw-semirings we study is finitely based (Sect. [3.3). All of these technical results rely on a study of properties of convex sets, filters and ideals in $\mathbb{Z}^{n}$ and $\mathbb{N}^{n}$ presented in the following section. 


\subsection{Convex Sets, Filters, and Ideals}

Suppose that $\bar{v}_{1}, \ldots, \bar{v}_{k}$ are vectors in $\mathbb{Z}^{n}$, or more generally, in $\mathbb{R}^{n}$. A convex linear combination of the $\bar{v}_{i}(i \in[k])$ is any vector $\bar{v} \in \mathbb{R}^{n}$ which can be written as

$$
\bar{v}=\lambda_{1} \bar{v}_{1}+\cdots+\lambda_{k} \bar{v}_{k}
$$

where $\lambda_{i} \geq 0, i \in[k]$, are real numbers with $\sum_{i=1}^{k} \lambda_{i}=1$.

Definition 2. Suppose that $U$ is any subset of $\mathbb{Z}^{n}$. We call $U$ a convex set if for all convex linear combinations $\bar{v}=\lambda_{1} \bar{v}_{1}+\cdots+\lambda_{k} \bar{v}_{k}$ with $k>0$ and $\bar{v}_{i} \in U$, $i \in[k]$, if $\bar{v} \in \mathbb{Z}^{n}$, then $\bar{v} \in U$.

Suppose that $U \subseteq \mathbb{N}^{n}$. We call $U$ an (order) ideal if for all $\bar{u}, \bar{v}$ in $\mathbb{N}^{n}$, if $\bar{u} \leq \bar{v}$ and $\bar{v} \in U$ then $\bar{u} \in U$. Moreover, we call $U$ a filter, if for all $\bar{u}$ and $\bar{v}$ as above, if $\bar{u} \in U$ then $\bar{v} \in U$. A convex ideal (respectively, convex filter) in $\mathbb{N}^{n}$ is any ideal (resp., filter) which is a convex set.

Note that order ideals and filters are sometimes referred to as lower and upper sets, respectively. The following fact is clear:

Proposition 1. The intersection of any number of convex sets in $\mathbb{Z}^{n}$ is convex. Moreover, the intersection of any number of convex ideals (convex filters) in $\mathbb{N}^{n}$ is a convex ideal (convex filter, respectively).

Thus each set $U \subseteq \mathbb{Z}^{n}$ is contained in a smallest convex set $[U]$ which is the intersection of all convex subsets of $\mathbb{Z}^{n}$ containing $U$. We call $[U]$ the convex set generated by $U$, or the convex hull of $U$. When $\bar{u} \in \mathbb{Z}^{n}$, below we shall sometimes write $[\bar{u}]$ for $[\{\bar{u}\}]=\{\bar{u}\}$.

Suppose now that $U \subseteq \mathbb{N}^{n}$. By Proposition 1, there is a smallest convex ideal $\operatorname{ci}(U)$ and a smallest convex filter $\operatorname{cf}(U)$ in $\mathbb{N}^{n}$ containing $U$. We call $\operatorname{ci}(U)$ and $\operatorname{cf}(U)$ the convex ideal and the convex filter generated by $U$, respectively.

For each set $U \subseteq \mathbb{N}^{n}$, define the ideal $(U]$ generated by $U$ thus:

$$
(U]=\left\{\bar{d} \in \mathbb{N}^{n}: \exists \bar{c} \in U . \bar{d} \leq \bar{c}\right\} .
$$

Similarly, the filter $[U)$ generated by $U$ is defined as:

$$
[U)=\left\{\bar{d} \in \mathbb{N}^{n}: \exists \bar{c} \in U . \bar{d} \geq \bar{c}\right\} .
$$

Throughout this study, we shall use $\bar{u}_{i}(i \in[n])$ to denote the $i$ th unit vector in $\mathbb{R}^{n}$, i.e., the vector whose only nonzero component is a 1 in the $i$ th position; $\overline{0}$ will denote the vector in $\mathbb{R}^{n}$ whose entries are all zero. When $\bar{u} \in \mathbb{N}^{n}$, below we shall sometimes write $(\bar{u}]$ and $[\bar{u})$ for $(\{\bar{u}\}]$ and $[\{\bar{u}\})$, respectively.

The following result, whose proof is nontrivial, plays an important role in the technical developments to follow.

Proposition 2. Suppose that $U \subseteq \mathbb{N}^{n}$. Then:

1. $\operatorname{ci}(U)=[(U]]$, and

2. $\operatorname{cf}(U)=[[U)]$. 
We can also show that $\operatorname{ci}(U)=\left([U]_{R}\right]$ and $\operatorname{cf}(U)=\left[[U]_{R}\right)$, where $[U]_{R}$ denotes the convex hull in $\mathbb{R}^{n}$ of the set $U$. However, the equalities $\operatorname{ci}(U)=([U]]$ and $\operatorname{cf}(U)=[[U])$ fail.

Henceforth, we shall use $[(U]]$ and $[[U)]$ to denote the convex ideal and the convex filter generated by $U$, respectively. This notation is justified by the above proposition.

Recall that a simple $\vee$-inequation in the variables $\bar{x}=\left(x_{1}, \ldots, x_{n}\right)$ is an inequation of the form

$$
\bar{d} \bar{x} \leq \bar{c}_{1} \bar{x} \vee \cdots \vee \bar{c}_{k} \bar{x}
$$

where $k>0$, and $\bar{d}, \bar{c}_{1}, \ldots, \bar{c}_{k} \in \mathbb{N}^{n}$. Similarly, a simple $\wedge$-inequation is of the form

$$
\bar{d} \bar{x} \geq \bar{c}_{1} \bar{x} \wedge \cdots \wedge \bar{c}_{k} \bar{x}
$$

where $k, \bar{d}$, and $\bar{c}_{i}(i \in[k])$ are as above. We recall that (11) holds in a ciw-semiring $\mathbf{A}_{\vee}=(A, \vee,+, 0)$ if the equation

$$
\bar{d} \bar{x} \vee \bar{c}_{1} \bar{x} \vee \cdots \vee \bar{c}_{k} \bar{x}=\bar{c}_{1} \bar{x} \vee \cdots \vee \bar{c}_{k} \bar{x}
$$

does, i.e., when for all $\bar{v} \in A^{n}$,

$$
\bar{d} \bar{v} \leq \bar{c}_{1} \bar{v} \vee \cdots \vee \bar{c}_{k} \bar{v}
$$

Similarly, we say that (2) holds in a ciw-semiring $\mathbf{A}_{\wedge}=(A, \wedge,+, 0)$ if the equation

$$
\bar{d} \bar{x} \wedge \bar{c}_{1} \bar{x} \wedge \cdots \wedge \bar{c}_{k} \bar{x}=\bar{c}_{1} \bar{x} \wedge \cdots \wedge \bar{c}_{k} \bar{x}
$$

does. Let $U$ denote the set $\left\{\bar{c}_{1}, \ldots, \bar{c}_{k}\right\}$. We recall that we shall sometimes abbreviate (1) as $\bar{d} \leq U$ and (2) as $\bar{d} \geq U$.

We now proceed to characterize the collection of simple inequations that hold in the algebra $\mathbf{Z}_{\vee}$ (and, thus, in its isomorphic version $\mathbf{Z}_{\wedge}$ ).

Proposition 3. Suppose that $\bar{d} \in \mathbb{N}^{n}$ and $U=\left\{\bar{c}_{1}, \ldots, \bar{c}_{k}\right\}$ is a finite nonempty set of vectors in $\mathbb{N}^{n}$. A simple inequation $\bar{d} \leq U$ holds in $\mathbf{Z}_{\vee}$ iff $\bar{d}$ belongs to the set $[U]$.

For $\mathbf{N}_{\vee}$ and $\mathbf{N}_{\wedge}$ we have:

Proposition 4. Suppose that $\bar{d} \in \mathbb{N}^{n}$ and $U$ is a finite nonempty set of vectors in $\mathbb{N}^{n}$.

1. The simple inequation $\overline{\bar{d}} \leq U$ holds in $\mathbf{N}_{\vee}$ iff $\bar{d}$ belongs to the set $[(U]]$.

2. The simple inequation $\bar{d} \geq U$ holds in $\mathbf{N}_{\wedge}$ iff $\bar{d}$ belongs to the set $[[U)]$.

The problem of deciding whether an equation holds in any of $\mathbf{Z}_{\vee}, \mathbf{N}_{\vee}$ and $\mathbf{N}_{\wedge}$ can be reduced to deciding whether a finite set of simple inequations holds in it (Cor. 1). The obvious reduction may result in a number of simple inequations that is exponential in the number of variables. However, using Propositions 3 and 4 the validity of a simple inequation in any of these structures can be tested in polynomial time by using linear programming (see, e.g., [19]). We therefore have that: 
Corollary 2. There exists an exponential time algorithm to decide whether an equation holds in the structures $\mathbf{Z}_{\vee}, \mathbf{N}_{\vee}$ and $\mathbf{N}_{\wedge}$. Moreover, it is decidable in polynomial time whether a simple inequation holds in these structures.

\subsection{Free Algebras and Relative Axiomatizations}

Let $C\left(\mathbb{N}^{n}\right), C I\left(\mathbb{N}^{n}\right)$ and $C F\left(\mathbb{N}^{n}\right)$ denote the sets of all finite nonempty convex sets, finite nonempty convex ideals, and nonempty convex filters in $\mathbb{N}^{n}$, respectively. We turn each of these sets into a ciw-semiring. Suppose that $U, V \in C\left(\mathbb{N}^{n}\right)$. First of all, recall that the complex sum of $U$ and $V$, notation $U \oplus V$, is defined thus:

$$
U \oplus V=\{\bar{u}+\bar{v}: \bar{u} \in U, \bar{v} \in V\} .
$$

We define

$$
\begin{aligned}
U \vee V & =[U \cup V] \\
U+V & =[U \oplus V] \\
0 & =[\overline{0}]=\{\overline{0}\} .
\end{aligned}
$$

We define the operations in $C I\left(\mathbb{N}^{n}\right)$ and $C F\left(\mathbb{N}^{n}\right)$ in a similar fashion. Suppose that $U, V \in C I\left(\mathbb{N}^{n}\right)$ and $U^{\prime}, V^{\prime} \in C F\left(\mathbb{N}^{n}\right)$. We set

$$
\begin{aligned}
U \vee V & =[(U \cup V]] \\
U+V & =[(U \oplus V]] \\
U^{\prime} \wedge V^{\prime} & =\left[\left[U^{\prime} \cup V^{\prime}\right)\right] \\
U^{\prime}+V^{\prime} & =\left[\left[U^{\prime} \oplus V^{\prime}\right)\right] .
\end{aligned}
$$

Moreover, we define $0=(\overline{0}]=\{\overline{0}\}$ in $C I\left(\mathbb{N}^{n}\right)$, and $0=[\overline{0})=\mathbb{N}^{n}$ in $C F\left(\mathbb{N}^{n}\right)$.

Proposition 5. Each of the structures $C\left(\mathbb{N}^{n}\right)=\left(C\left(\mathbb{N}^{n}\right), \vee,+, 0\right), C I\left(\mathbb{N}^{n}\right)=$ $\left(C I\left(\mathbb{N}^{n}\right), \vee,+, 0\right)$ and $C F\left(\mathbb{N}^{n}\right)=\left(C F\left(\mathbb{N}^{n}\right), \wedge,+, 0\right)$ is a ciw-semiring. In addition, $C I\left(\mathbb{N}^{n}\right)$ satisfies the equation

$$
x \vee 0=x,
$$

and $C F\left(\mathbb{N}^{n}\right)$ the equation

$$
x \wedge 0=0 .
$$

Note that (3) can be rephrased, with respect to $E_{\text {ciw }}$, as the inequation $0 \leq x$, and (4) as $x \geq 0$. Also, writing $\vee$ for $\wedge$, (4) takes the form $x \vee 0=0$ that one should have if $\vee$ is considered to be the signature symbol instead of $\wedge$.

For any structure $\mathbf{A}$, we use $\mathcal{V}(\mathbf{A})$ to denote the variety generated by $\mathbf{A}$, i.e., the class of algebras that satisfy the equations that hold in $\mathbf{A}$. 
Theorem 1. For each $n \geq 0$,

1. $C\left(\mathbb{N}^{n}\right)$ is freely generated in $\mathcal{V}\left(\mathbf{Z}_{\vee}\right)$ by the sets $\left[\bar{u}_{i}\right], i \in[n]$,

2. $C I\left(\mathbb{N}^{n}\right)$ is freely generated in $\mathcal{V}\left(\mathbf{N}_{\vee}\right)$ by the sets $\left(\bar{u}_{i}\right], i \in[n]$, and

3. $C F\left(\mathbb{N}^{n}\right)$ is freely generated in $\mathcal{V}\left(\mathbf{N}_{\wedge}\right)$ by the sets $\left[\bar{u}_{i}\right), i \in[n]$.

Remark 2. In any variety, any infinitely generated free algebra is the direct limit of finitely generated free algebras. More specifically, for any cardinal number $\kappa$, the free algebra on $\kappa$ generators in $\mathcal{V}\left(\mathbf{Z}_{\vee}\right)$ can be described as an algebra of finite nonempty convex sets in $\mathbb{N}^{\kappa}$ consisting of vectors whose components, with a finite number of exceptions, are all zero. The free algebras in $\mathcal{V}\left(\mathbf{N}_{\vee}\right)$ and $\mathcal{V}\left(\mathbf{N}_{\wedge}\right)$ have similar descriptions using finitely generated convex ideals and filters, respectively.

Since $\mathbf{N}_{\vee}$ is a subalgebra of $\mathbf{Z}_{\vee}$, we have that $\mathcal{V}\left(\mathbf{N}_{\vee}\right) \subseteq \mathcal{V}\left(\mathbf{Z}_{\vee}\right)$. Also, since $\mathbf{N}_{\wedge}$ is isomorphic to the subalgebra $\mathbf{N}_{\vee}^{-}$of $\mathbf{Z}_{\vee}$, it holds that $\mathcal{V}\left(\mathbf{N}_{\wedge}\right) \subseteq \mathcal{V}\left(\mathbf{Z}_{\vee}\right)$.

Definition 3. Let $\mathcal{V}$ and $\mathcal{V}^{\prime}$ be two varieties of algebras over the same signature $\Sigma$. Let $E$ be a collection of equations over $\Sigma$. We say that $\mathcal{V}$ is axiomatized over $\mathcal{V}^{\prime}$ by $E$ if the collection of equations that hold in $\mathcal{V}^{\prime}$ together with $E$ form a basis for the identities of $\mathcal{V}$. We say that $\mathcal{V}$ has a finite axiomatization relative to $\mathcal{V}^{\prime}$ if $\mathcal{V}$ is axiomatized over $\mathcal{V}^{\prime}$ by some finite set of equations $E$.

In the next result we show that both $\mathcal{V}\left(\mathbf{N}_{\vee}\right)$ and $\mathcal{V}\left(\mathbf{N}_{\wedge}\right)$ possess a finite axiomatization relative to $\mathcal{V}\left(\mathbf{Z}_{\vee}\right)$. Of course, $\mathcal{V}\left(\mathbf{Z}_{\vee}\right)$ is just $\mathcal{V}\left(\mathbf{Z}_{\wedge}\right)$, since $\mathbf{Z}_{\vee}$ and $\mathbf{Z}_{\wedge}$ are also isomorphic.

\section{Theorem 2.}

1. $\mathcal{V}\left(\mathbf{N}_{\vee}\right)$ is axiomatized over $\mathcal{V}\left(\mathbf{Z}_{\vee}\right)$ by the equation (3).

2. $\mathcal{V}\left(\mathbf{N}_{\wedge}\right)$ is axiomatized over $\mathcal{V}\left(\mathbf{Z}_{\wedge}\right)$ by the equation (4).

\subsection{Non-finite Axiomatizability Results}

Our order of business in this section is to show that the varieties generated by the ciw-semirings $\mathbf{Z}_{\vee}$ and $\mathbf{N}_{\wedge}$ are not finitely based. Our starting points are the results in $\left[1 \mid 2\right.$ to the effect that the variety $\mathcal{V}\left(\mathbf{N}_{\vee}\right)$ is not finitely based. By using these results, and the first part of Theorem 2 , we can derive:

Theorem 3. The variety $\mathcal{V}\left(\mathbf{Z}_{\vee}\right)$ is not finitely based. Moreover, there exists no natural number $n$ such that the collection of all equations in at most $n$ variables that hold in $\mathcal{V}\left(\mathbf{Z}_{\vee}\right)$ forms an equational basis for $\mathcal{V}\left(\mathbf{Z}_{\vee}\right)$. Finally, the collection of equations in two variables that hold in the variety $\mathcal{V}\left(\mathbf{Z}_{\vee}\right)$ has no finite equational axiomatization.

Our aim in the remainder of this section is to outline a proof of the following result to the effect that the variety $\mathcal{V}\left(\mathbf{N}_{\wedge}\right)$ has no finite equational basis.

Theorem 4. The variety $\mathcal{V}\left(\mathbf{N}_{\wedge}\right)$ has no finite (equational) axiomatization.

In fact, the above theorem is a consequence of the following stronger result. 
Theorem 5. There exists no natural number $n$ such that the collection of all equations in at most $n$ variables that hold in $\mathcal{V}\left(\mathbf{N}_{\wedge}\right)$ forms an equational basis for $\mathcal{V}\left(\mathbf{N}_{\wedge}\right)$.

To prove Theorem [5, we begin by noting that the following equations $e_{n}$ hold in $\mathbf{N}_{\wedge}$, for each $n \geq 2$ :

$$
e_{n}: \quad r_{n} \wedge s_{n}=s_{n}
$$

where

$$
\begin{aligned}
r_{n}= & x_{1}+\cdots+x_{n} \\
s_{n}= & \left(2 x_{1}+x_{3}+x_{4}+\cdots+x_{n-1}+x_{n}\right) \\
& \wedge\left(x_{1}+2 x_{2}+x_{4}+\cdots+x_{n-1}+x_{n}\right) \\
& \vdots \\
& \wedge\left(x_{1}+x_{2}+x_{3}+\cdots+x_{n-2}+2 x_{n-1}\right) \\
& \wedge\left(x_{2}+x_{3}+x_{4}+\cdots+x_{n-1}+2 x_{n}\right) .
\end{aligned}
$$

We then define a sequence of countably infinite ciw-semirings $\mathbf{B}_{n}(n \geq 3)$ which are a model of all the equations in at most $n-1$ variables that hold in $\mathbf{N}_{\wedge}$, but in which $e_{n}$ fails. The overall proof strategy is similar to the one we used to prove the main result in [1], but the actual technical details of the proof are significantly different.

We introduce the following notation for some vectors in $\mathbb{N}^{n}$ related to the equation $e_{n}$ :

$$
\begin{aligned}
\bar{\delta} & =(1, \ldots, 1) \\
\bar{\gamma}_{1} & =(2,0,1,1, \ldots, 1,1) \\
\bar{\gamma}_{2} & =(1,2,0,1, \ldots, 1,1) \\
& \vdots \\
\bar{\gamma}_{n-1} & =(1,1,1,1, \ldots, 2,0) \\
\bar{\gamma}_{n} & =(0,1,1,1, \ldots, 1,2),
\end{aligned}
$$

so that in $\bar{\gamma}_{i}(i \in[n])$, the 2 is on the $i$ th position and is followed by a 0 . (Of course, we assume that the first position follows the $n$ th.) All other components are 1's. Note that

$$
\bar{\delta}=\frac{1}{n} \bar{\gamma}_{1}+\cdots+\frac{1}{n} \bar{\gamma}_{n}
$$

Thus, $\bar{\delta}$ belongs to the convex filter generated by the vectors $\bar{\gamma}_{i}(i \in[n])$. Moreover, the system consisting of any $n$ of the vectors $\bar{\delta}, \bar{\gamma}_{1}, \ldots, \bar{\gamma}_{n}$ is linearly independent.

We define:

$$
\begin{aligned}
\Gamma & =\left[\left[\left\{\bar{\gamma}_{1}, \ldots, \bar{\gamma}_{n}\right\}\right)\right] \\
\Delta & =\Gamma-\{\bar{\delta}\}
\end{aligned}
$$


so that $\Gamma$ is the convex filter generated by the $\bar{\gamma}_{i}(i \in[n])$. By [6] , the set $\Delta$ is not a convex filter.

We now proceed to define the algebras $\mathbf{B}_{n}=\left(B_{n}, \wedge,+, 0\right), n>0$. Let $B_{n}$ consist of the nonempty convex filters in $\mathbb{N}^{n}$ and the set $\Delta$.

Proposition 6. If the intersection of a family of sets in $B_{n}$ is not empty, then the intersection is in $B_{n}$.

For each nonempty set $U \subseteq \mathbb{N}^{n}$, let $\operatorname{cl}(U)$ denote the least set in $B_{n}$ containing $U$. For each $U, V \in B_{n}$, we define

$$
\begin{aligned}
& U+V=\operatorname{cl}(U \oplus V) \\
& U \wedge V=\operatorname{cl}(U \cup V) .
\end{aligned}
$$

Moreover, we define the constant 0 to be the set $[[0)]=[0)=\mathbb{N}^{n}$. This completes the definition of the algebra $\mathbf{B}_{n}=\left(B_{n}, \wedge,+, 0\right)$.

Proposition 7. If $n \geq 3$, then $\mathbf{B}_{n}$ is a ciw-semiring satisfying $x \wedge 0=0$ but not satisfying $e_{n}$.

Indeed, for each $i \in[n]$, let $\bar{u}_{i}$ denote the $i$ th $n$-dimensional unit vector whose components are all 0 except for a 1 in the $i$ th position. We have

$$
\begin{aligned}
& r_{n}\left(\left[\left[\bar{u}_{1}\right)\right], \ldots,\left[\left[\bar{u}_{n}\right)\right]\right)=[[\bar{\delta})] \text { and } \\
& s_{n}\left(\left[\left[\bar{u}_{1}\right)\right], \ldots,\left[\left[\bar{u}_{n}\right)\right]\right)=\Delta
\end{aligned}
$$

in $\mathbf{B}_{n}$.

Proposition 8. For each $n \geq 3$, the algebra $\mathbf{B}_{n}$ satisfies any equation in at most $n-1$ variables which holds in $\mathbf{N}_{\wedge}$.

Theorem [5] is an immediate consequence of the above facts.

\section{Tropical Semirings}

We now proceed to investigate the equational theory of the tropical semirings studied in the literature that are obtained by adding bottom elements to the ciw-semirings presented in the previous section. More specifically, we shall study the following semirings:

$$
\begin{aligned}
\mathbf{Z}_{\vee,-\infty} & =(\mathbb{Z} \cup\{-\infty\}, \vee,+,-\infty, 0), \\
\mathbf{N}_{\vee,-\infty} & =(\mathbb{N} \cup\{-\infty\}, \vee,+,-\infty, 0) \text { and } \\
\mathbf{N}_{\vee,-\infty}^{-} & =\left(\mathbb{N}^{-} \cup\{-\infty\}, \vee,+,-\infty, 0\right) .
\end{aligned}
$$

Since $\mathbf{Z}_{\vee,-\infty}$ and $\mathbf{N}_{\vee,-\infty}^{-}$are isomorphic to $\mathbf{Z}_{\wedge, \infty}=(\mathbb{Z} \cup\{\infty\}, \wedge,+, \infty, 0)$ and $\mathbf{N}_{\wedge, \infty}=(\mathbb{N} \cup\{\infty\}, \wedge,+, \infty, 0)$, respectively, the results that we shall obtain apply equally well to these algebras. In fact, rather than studying the algebra 
$\mathbf{N}_{\vee,-\infty}^{-}$, we shall work with the semiring $\mathbf{N}_{\wedge, \infty}$. (The semirings $\mathbf{Z}_{\wedge, \infty}$ and $\mathbf{N}_{\wedge, \infty}$ are usually referred to as the equatorial semiring [15] and the tropical semiring [20], respectively. The semiring $\mathbf{N}_{\vee,-\infty}$ is called the polar semiring in [17].)

In Sect. 2, we saw how to generate a commutative idempotent semiring $\mathbf{A}_{\perp}=\left(A_{\perp}, \vee,+, \perp, 0\right)$ from any ciw-semiring $\mathbf{A}=(A, \vee,+, 0)$ by freely adding a bottom element $\perp$ to it. We now proceed to study some general relationships between the equational theories of these two structures. The results that we shall obtain are the keys to obtain non-finite axiomatizability results for the above tropical semirings associated with the ciw-semirings.

Definition 4. An inequation is strictly regular if its two sides contain the same variables.

Let $\mathbf{A} \in \mathcal{V}_{\text {ciw. }}$. We say that $\mathbf{A}$ is strictly regularly based if every simple inequation that holds in it is provable, modulo $E_{\text {ciw }}$, from a strictly regular one that holds in $\mathbf{A}$.

The following general result will allow us to lift non-finite axiomatizability results from some ciw-semirings to the commutative idempotent semirings they underlie.

Proposition 9. Suppose that $\mathbf{A} \in \mathcal{V}_{\text {ciw }}$ is strictly regularly based. If $\mathcal{V}(\mathbf{A})$ has no finite axiomatization, then $\mathcal{V}\left(\mathbf{A}_{\perp}\right)$ has no finite axiomatization either.

\section{Theorem 6.}

1. The ciw-semirings $\mathbf{Z}_{\vee}$ and $\mathbf{N}_{\vee}$ are strictly regularly based.

2. The varieties $\mathcal{V}\left(\mathbf{Z}_{\vee,-\infty}\right)$ and $\mathcal{V}\left(\mathbf{N}_{\vee,-\infty}\right)$ are not finitely based.

We now relate the equational theory of the original tropical semiring $\mathbf{N}_{\wedge, \infty}=$ $(\mathbb{N} \cup\{\infty\}, \wedge,+, \infty, 0)$ from [20] to the equational theory of the algebra $\mathbf{N}_{\wedge}=$ $(\mathbb{N}, \wedge,+, 0)$. For the sake of clarity, we recall that + is standard addition (extended to $\mathbb{N} \cup\{\infty\}$ by stipulating that $\infty+x=x+\infty=\infty)$.

The structures $\mathbf{N}_{\wedge}$ and $\mathbf{N}_{\wedge, \infty}$ satisfy the same equations in the language of $\mathbf{N}_{\wedge}$. This is the import of the following lemma.

Lemma 3. Let $t \geq t^{\prime}$ be a simple $\wedge$-inequation in the language of $\mathbf{N}_{\wedge}$. Then $t \geq t^{\prime}$ holds in $\mathbf{N}_{\wedge}$ iff it holds in $\mathbf{N}_{\wedge, \infty}$.

As a corollary of this result, we have that:

Theorem 7. The varieties $\mathcal{V}\left(\mathbf{N}_{\wedge, \infty}\right)$ and $\mathcal{V}\left(\mathbf{N}_{\vee,-\infty}^{-}\right)$have no finite axiomatization.

\section{$5 \quad$ Further Results}

In this section, we outline some further results. For details, the reader is referred to the extended abstract [3] and the technical report [4].

We have shown that none of the tropical semirings $\mathbf{Z}_{\vee,-\infty}, \mathbf{N}_{\vee,-\infty}$ and $\mathbf{N}_{\vee,-\infty}^{-}$, or $\mathbf{N}_{\wedge, \infty}$, has a finite basis for its equations. But what is a complete 
description of the equations, or rather, simple inequations that hold in these semirings? As regards $\mathbf{N}_{\wedge, \infty}$, we have already obtained a satisfactory answer. As for $\mathbf{Z}_{\vee,-\infty}$, it can be seen easily that a simple inequation holds in $\mathbf{Z}_{\vee,-\infty}$ if and only if it holds in $\mathbf{Z}_{\vee}$. The case of $\mathbf{N}_{\vee,-\infty}$ is more challenging. Suppose that $U$ is a finite nonempty subset of $\mathbb{N}^{n}$ and $\bar{d} \in U$. Then we have that a simple inequation $\bar{d} \leq U$ holds in $\mathbf{N}_{\vee,-\infty}$ iff $\bar{d} \in \mathrm{ci}^{+}(U)$, where $\mathrm{ci}^{+}(U)$ denotes the positive convex ideal 34 generated by $U$. This characterization of the valid inequations has three corollaries. First, it follows that an algebra of the positive convex ideals in $\mathbb{N}^{n}$ can be characterized as the $n$-generated free algebra in $\mathcal{V}\left(\mathbf{N}_{\vee},-\infty\right)$. Second, it follows that $\mathcal{V}\left(\mathbf{N}_{\vee,-\infty}\right)$ can be axiomatized over $\mathcal{V}\left(\mathbf{Z}_{\vee,-\infty}\right)$ by the simple inequation $x \leq x+x$. Third, it follows that the equational theory of $\mathcal{V}\left(\mathbf{N}_{\vee,-\infty}\right)$ is decidable in exponential time.

There are some further variations. These include structures based on the rational or real numbers, or just the nonnegative on nonpositive rational or real numbers. Semirings involving rationals or reals always have the same equational theory as the subalgebra of the integers included in the semiring. Thus, e.g., the semiring $\mathbf{Q}_{\vee,-\infty}=(\mathbb{Q} \cup\{-\infty\}, \vee,+,-\infty, 0)$ has the same equational theory as the semiring $\mathbf{Z}_{\vee,-\infty}$, and the equational theory of $\mathbf{Q}_{\vee,-\infty}^{+}=$ $\left(\mathbb{Q}^{+} \cup\{-\infty\}, \vee,+,-\infty, 0\right)$ agrees with that of $\mathbf{N}_{\vee,-\infty}$.

Acknowledgement. We would like to thank the anonymous referees whose suggestions helped us improve the paper.

\section{References}

1. L. ACETo, Z. ÉSIK, AND A. IngólfsDótTiR, The max-plus algebra of the natural numbers has no finite equational basis, BRICS Report RS-99-33, October 1999. To appear in Theoretical Computer Science.

2. - On the two-variable fragment of the equational theory of the max-sum algebra of the natural numbers, in Proceedings of the 17th STACS, H. Reichel and S. Tison, eds., vol. 1770 of Lecture Notes in Computer Science, Springer-Verlag, Feb. 2000, pp. 267-278.

3. — Nonfinitely based tropical semirings, in Proceedings of the Int. Workshop on Max-Plus Algebras, Praha, August, 2001, to appear.

4. — Axiomatizing tropical semirings, to appear as BRICS research report.

5. F. Baccelli, G. Cohen, G.-J. Olsder, And J.-P. Quadrat, Synchronization and Linearity, Wiley Series in Probability and Mathematical Statistics, John Wiley, 1992.

6. A. Bonnier-Rigny And D. Krob, A complete system of identities for one-letter rational expressions with multiplicities in the tropical semiring, Theoretical Computer Science, 134 (1994), pp. 27-50.

7. W. BuRnside, On an unsettled question in the theory of discontinuous groups, Q. J. Pure and Appl. Mathematics, 33 (1902), pp. 230-238.

8. S. Burris and H. P. Sankappanavar, A Course in Universal Algebra, SpringerVerlag, New York, 1981.

9. J. H. Conway, Regular Algebra and Finite Machines, Mathematics Series (R. Brown and J. De Wet eds.), Chapman and Hall, London, United Kingdom, 1971. 
10. J. Gunawardena (ED.), Idempotency, Publications of the Newton Institute 11, Cambridge University Press, 1998.

11. —, An introduction to idempotency, in [10], pp. 1-49.

12. K. HASHIGUCHI, Limitedness theorem on finite automata with distance functions, J. Comput. System Sci., 24 (1982), no. 2, pp. 233-244.

13. - Regular languages of star height one, Information and Control, 53 (1982), pp. 199-210.

14. - Algorithms for determining relative star height and star height, Information and Computation, 78 (1988), pp. 124-169.

15. D. Квов, The equality problem for rational series with multiplicities in the tropical semiring is undecidable, International J. Algebra Computation, 4 (1994), pp. 405425.

16. - Some consequences of a Fatou property of the tropical semiring, Journal of Pure and Applied Algebra, 93 (1994), pp. 231-249.

17. — Some automata-theoretic aspects of min-max-plus semirings, in [10], pp. 7079 .

18. J.-E. PIN, Tropical semirings, in [10, pp. 50-69.

19. A. SchriJver, Theory of Linear and Integer Programming, John Wiley and Sons, 1986.

20. I. Simon, Limited subsets of a free monoid, in Proceedings of the 19th Annual Symposium on Foundations of Computer Science (Ann Arbor, Mich., 1978), IEEE Computer Society, 1978, pp. 143-150.

21. — The nondeterministic complexity of finite automata, in Mots, M. Lothaire ed., Hermès, 1990, pp. 384-400.

22. U. Zimmermann, Linear and Combinatorial Optimization in Ordered Algebraic Structures, Annals of Discrete Mathematics 10, North-Holland, 1981. 